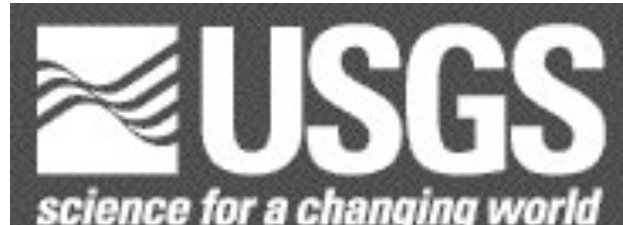

\title{
SEGY to ASCII Conversion and Plotting Program 2.0
}

By Mark. R. Goldman 1

Open-File Report 2005-1311

This Open-File Report supersedes USGS Open-File Repor99-126

This document and any updates to it may be found at:

http://pubs.usgs.gov/of/2005/1311/

Any use of trade, firm, or product names is for descriptive purposes only and does not imply endorsement by the U.S. Government.

U.S. DEPARTMENT OF THE INTERIOR

U.S. GEOLOGICAL SURVEY

${ }^{1}$ Menlo Park, Calif. 


\section{CONTENTS AND PROGRAM REQUIREMENTS}

Date of Latest Revision: July, 2005

U.S. Department of the Interior

U.S. Geological Survey

Open-File Report 2005-1311

SEGY to ASCII Conversion and Plotting Package 2.0

Mark Goldman

U.S. Geological Survey

MS 977

345 Middlefield Rd.

Menlo Park, CA 94025

Although this program has been used by the U.S. Geological Survey, no warranty, expressed or implied, is made by the USGS as to the accuracy and functioning of the program and related program material, nor shall the fact of distribution constitute any such warranty, and no responsibility is assumed by the USGS in connection therewith.

This directory contains the source code and shell scripts necessary to convert SEGY files to ASCII format and to create postscript plots, along with a makefile and a README file. This directory also contains a subdirectory with an example SEGY file, ASCII file, shell script file, and resulting postscript file. As a test, one can run the shell script file to recreate the xyz and postscript files.

The shell scripts run using the csh shell command interpreter, which is present on most Unix systems.

A requirement for compiling the source code is a $\mathrm{C}++$ compiler The $\mathrm{C}++$ programs documented here have been successfully compiled using Gnu's g++ version 3.3.2. The g++ compiler is a free $\mathrm{C}++$ compiler and may be downloaded from the web site:

http://gcc.gnu.org

A requirement for plotting the seismic data is the GMT plotting package. The shell scripts documented here have successfully produced postscript files using Gmt version 3.3.4. The GMT plotting package is a free set of plotting routines, and may be downloaded from the web site: http://gmt.soest.hawaii.edu 


\section{INTRODUCTION}

SEGY has long been a standard format for storing seismic data and header information. Almost every seismic processing package can read and write seismic data in SEGY format. In the data processing world, however, ASCII format is the "universal" standard format. Very few general purpose plotting or computation programs will accept data in SEGY format.

The software presented in this report, referred to as SEGY to ASCII (SAC), converts seismic data written in SEGY format (Barry et al., 1975) to an ASCII data file, and then creates a postscript file of the seismic data using a general plotting package (GMT, Wessel and Smith, 1995). The resulting postscript file may be plotted by any standard postscript plotting program.

There are two versions of SAC: one version for plotting a SEGY file that contains a single gather, such as a stacked CDP or migrated section, and a second version for plotting multiple gathers from a SEGY file containing more than one gather, such as a collection of shot gathers. Note that if a SEGY file has multiple gathers, then each gather must have the same number of traces per gather, and each trace must have the same sample interval and number of samples per trace.

SAC will read several common standards of SEGY data, including SEGY files with sample values written in either IBM or IEEE floating point format. In addition, utility programs are present to convert non-standard Seismic Unix (.sux) SEGY files and PASSCAL (.rsy) SEGY files to standard SEGY files.

SAC allows complete user control over all plotting parameters including label size and font, tick mark intervals, trace scaling, and the inclusion of a title and descriptive text.

SAC shell scripts create a postscript image of the seismic data in vector rather than bitmap format, using GMT's pswiggle command. Although this can produce a very large postscript file, the image quality is generally superior to that of a bitmap image, and commercial programs such as Adobe Illustrator ${ }^{\circledR}$ can manipulate the image more efficiently. 


\begin{tabular}{|c|c|}
\hline PROGRAM NAME & DESCRIPTION \\
\hline README.txt & this file as a text document \\
\hline README.doc & this file as a Microsoft Word document \\
\hline makefile & makefile for use in compilation \\
\hline $\begin{array}{l}\text { splot } \\
\text { splot2 }\end{array}$ & $\begin{array}{l}\text { template csh shell script for plotting a single gather } \\
\text { template csh shell script for plotting multiple gathers }\end{array}$ \\
\hline splot.body & csh shell script called by splot (do not edit) \\
\hline splot2.body & csh shell script called by splot2 (do not edit) \\
\hline $\operatorname{segy} 2 x y z$ & $\begin{array}{l}\text { program to convert a SEGY file to ASCII, } \\
\text { called by shell script }\end{array}$ \\
\hline segy $2 x y z 2$ & $\begin{array}{l}\text { program to convert a particular gather within a } \\
\text { multi-gather SEGY file to ASCII, called by shell script }\end{array}$ \\
\hline segyheader & utility to list a SEGY file's header values \\
\hline $\begin{array}{l}\text { segyibm2ieee } \\
\text { su2segy }\end{array}$ & $\begin{array}{l}\text { utility to convert sample values from IBM to IEEE } \\
\text { utility to combine several Seismic Unix (.sux) } \\
\text { SEGY files into a single SEGY file }\end{array}$ \\
\hline rsy2segy & $\begin{array}{l}\text { utility to combine several Passcal (.rsy) } \\
\text { SEGY files into a single SEGY file }\end{array}$ \\
\hline xyz2segy & utility to convert xyz data to SEGY format \\
\hline $\begin{array}{l}\text { testEndian } \\
\text { segyswap }\end{array}$ & $\begin{array}{l}\text { utility to determine if your computer's is big or little Endian } \\
\text { utility to convert a SEGY file between big/little Endian }\end{array}$ \\
\hline \multicolumn{2}{|c|}{ In Example Subdirectory: } \\
\hline $\begin{array}{l}\text { README } \\
\text { example } \\
\text { example.segy } \\
\text { example.xyz } \\
\text { example.1000wv.ps }\end{array}$ & $\begin{array}{l}\text { readme file for this directory } \\
\text { edited copy of the splot template shell script } \\
\text { input SEGY file of a stacked depth section } \\
\text { output ASCII file } \\
\text { output postscript file, } 1000 \mathrm{~ms} \text { depth, } \\
\text { combined wiggle trace and variable area display } \\
\text { cdn VS x coordinate file }\end{array}$ \\
\hline
\end{tabular}




\section{INPUT DATA FORMAT}

SAC reads standard 4 byte IBM or IEEE floating point SEGY files, reading the number of samples, sample rate, channel, and common depth point values directly from the SEGY file.

SAC assumes that the input SEGY file starts with a 3600-byte reel header, with bytes 3217-3218 containing the sample rate in microseconds and bytes 3221-3222 containing the number of samples.

Each trace should be preceded by a 240-byte trace ID header, with bytes $13-16$ containing the channel number and for a cdp gather, bytes 21-24 containing the cdp for the trace.

SEGY files may contain more than one gather per trace, though this is non-standard. For example, a SEGY files will often contains several shot gathers. For SAC to run, each gather must have the same number of traces per gather, and each trace must have the same sample interval and number of samples.

A common problem when dealing with binary formats like SEGY is the byte order, which can be either big-Endian (on Mac's and Unix machines) or little-Endian format (on PC's). A SEGY file created on a little-Endian machine will look like garbage if read on a big-Endian machine, and visa-versa. A utility program, segyswap, is included to toggle the SEGY file between these two types. If you encounter an "unexpected end of SEGY file" error message, or the output looks like garbage, try swapping the byte order of the SEGY file. 


\section{OUTPUT DATA FORMAT}

SAC converts an input SEGY file to an ASCII file in xyz format, where $\mathrm{x}$ is the channel number, CDP, or the $\mathrm{x}$ coordinate of the trace, $\mathrm{y}$ is the time or depth of the trace sample, and $\mathrm{z}$ is the amplitude of the trace sample.

A line containing only the '\#' character in column 1 precedes the listing for each trace. After this line the trace samples are listed, one sample per line, with the $\mathrm{x}, \mathrm{y}$, and $\mathrm{z}$ values of the sample separated by spaces or tabs.

Samples are listed in order of ascending time or depth. All values are right justified within specific column ranges. The $\mathrm{x}$ value is in columns $1-8$, the $\mathrm{y}$ value in columns $10-17$, and the $\mathrm{z}$ value in columns 19-30.

Below is an example for 2 traces (channels 1 and 2), each with 2 samples per trace (sample times of 0 and $5 \mathrm{~ms}$ ).

$\begin{array}{llll}\text { \# } & & & \\ & & & \\ & 1.00 & 0 & -1.6961 \\ 1.00 & 5 & -0.1384 \\ & & & \\ & 2.00 & 0 & 0.1334 \\ & 2.00 & 5 & 1.9543\end{array}$

\section{CDP CONVERSION FILE FORMAT}

The CDP to $\mathrm{x}$ coordinate conversion file has a free-form format, where the first field is the CDP number, and the second field is the x coordinate. Header and comment lines are not necessary, but may be included if they start with a '\#' character in column 1.

Below is an example for a typical CDP to x coordinate conversion file.

\# optional comment line

197.2345

$2 \quad 100.1256$ 


\section{GETTING STARTED}

Move to the directory containing the SAC code and this README file.

Compile the $\mathrm{C}++$ source code using the makefile provided by typing "make all".

Create a working subdirectory within the SAC directory, and copy the splot or splot 2 template shell script to your working directory, perhaps renaming the shell script to a more descriptive name within your working directory (see the Example subdirectory).

Move to your working directory

Edit your working directory's version of the template shell script, and set the conversion and plotting parameters within that file.

note: do not edit the template splot or splot shell scripts in the main SAC directory, only edit copies of these shell scripts in your own working directory

Move the input SEGY file to your working directory. The SEGY file must have the suffix ".segy"

note: if using FTP, make sure you set the transfer type to "binary"

If needed, create a cdp to x coordinate conversion file in your working directory.

Run the shell script in your working directory by typing the name of the shell script, followed by the name of the input SEGY file. This should create an ASCII xyz file and a postscript file.

The postscript file will have the suffix "xy.ps", where "x" is the maximum time or depth, and "y" is the trace display type ( $\mathrm{c}$ for grayscale display, w for wiggle display, $\mathrm{v}$ for variable area display). For example, a postscript plot of a gather down to $500 \mathrm{~ms}$, with wiggle and variable area trace display, will have the suffix "500wv.ps"

If plotting multiple gathers using the splot 2 shell script, then the postscript file will have the suffix "gather.xy.ps", where "gather" is the gather number + offset, with offset defined in the splot 2 shell script. The gather number is the sequential gather in the SEGY file, starting at one and incrementing by one with every subsequent gather. 


\begin{tabular}{|c|c|c|}
\hline \# SEISMIC PI & T PEN V & TH AND COLOR ATTRIBUTES \\
\hline set fill_color & $=0$ & \# 0-255 grayscale OR red/green/blue format \\
\hline set plot_color & $=0$ & \# 0-255 grayscale OR red/green/blue format \\
\hline set plot_pen & $=0.1$ & \# pen width/style for seismic traces \\
\hline set ft_pen & $=5$ & \# pen width/style for frame and tick marks \\
\hline set tick_ln & $=0.12$ & \# tick length \\
\hline set anot_off & $=0.06$ & \# offset of annotation from frame \\
\hline \# OFFSETS S & ARATINC & IMAGE OBJECTS (IN INCHES) \\
\hline set title_text_o & $=0.25$ & \# offset between title and text 1 \\
\hline set text1_text2 & $\mathrm{ff}=0.20$ & \# offset between text 1 and text 2 \\
\hline set text_plot_o & $=0.70$ & \# offset between text and seismic plot \\
\hline
\end{tabular}

\section{PASSCAL SEGY DEVIATIONS FROM STANDARD SEGY:}

1) missing initial 3600 byte reel header

2) number of samples are at bytes 229-232 (allows for higher values than 32767)

3) sample values are in 2 or 4 byte integer format

(bytes $205-206=0$ then 2 byte integer format, $=1$ then 4 byte integer format)

4) bytes 221-224 contain a floating point scaling factor, where true value $=$ sample value $*$ scaling factor

5) bytes 225-226 have integer DAS instrument number $+10,000$

\section{SEISMIC UNIX SEGY DEVIATIONS FROM STANDARD SEGY:}

1) missing initial 3600 byte reel header

2) number of samples is still at bytes $115-166$ (limited to 32767 samples/trace)

3) sample values are in 4 byte IEEE floating point format

4) bytes $175-176$ have integer DAS instrument number $+10,000$

\section{REFERENCES}

Wessel, P., and W.H.F. Smith, 1995, The Generic Mapping Tools (GMT) version 3.0 Technical Reference \& Cookbook, SOEST/NOAA.

Barry, K.M., Cavers, D.A., and Kneal, C.W., 1975, Recommended Standards for Digital Tape Format, Geophysics, vol 40, no 2, p. 344-352. 